\title{
The Generalized Form of Principia's Third Law of Motion
}

\author{
Ajay Sharma* \\ Fundamental Physics Society His Mercy Enclave, Post Box 107 GPO, Shimla - 171001, Himachal Pradesh, \\ India; ajoy.plus@gmail.com
}

\begin{abstract}
Objective: Third law is critically analysed in view of definition and elaboration given by Newton at page 20 of the Principia. It is evident that law is incompletely studied. Methods/Analysis: Newton did not give any mathematical equation for the law. The definition and explanation of the law is completely analyzed theoretically and discussed in view of the experimental data. Newton's explanation is simply qualitative, not quantitative which is required for scientific analysis. The law is universally applicable to all cases and all bodies without any constraint. However Newton elaborated the same in terms of just three qualitative examples which is not scientific. Findings: The terms action and reaction neither possess units nor dimensions, even expressed in terms of equations the explanation is qualitative only. The law is understood in terms of force only. However Newton expressed law in terms of both force and velocity in three examples. If law is expressed in terms of force and velocity analogous to action and reaction, then its limitations are evident. For example it does not account for the significant factors e.g. inherent characteristics, nature, compositions, flexibility, rigidity, magnitude, size, elasticity, shape, distinctiveness of interacting bodies, mode of interactions, point of impact etc. The law is universally applicable for all bodies e.g. bodies may be of steel, wood, rubber, cloth, wool, sponge, spring, typical plastic, porous material, air/fluid filled artifact, mud or kneaded flour or chewing gum specifically fabricated material etc. For all such bodies if the action is same, then the reaction must be the same. But it is not found true in all cases, as interaction between rubber and steel are different from rubber and cotton. Application/Improvements: Thus law is generalized; reaction is proportional to action depending upon characteristics of system. Mathematically, Reaction = $-\mathrm{K}$ action, the value of $\mathrm{K}$ may be equal to, less than, or greater than unity depending upon experimental parameters.
\end{abstract}

Keywords: Coefficient, Generalized, Newton, Third Law

\section{The Principia's Third Law of Motion}

The original form of the Third Law of Motion is:

To every action there is always opposed an equal reaction; or the mutual actions of two bodies upon each other are always equal, and directed to contrary parts"]

Action $=-$ Reaction

or Mutual action of one body $=-$ Mutual reaction of other body

There is no other factor in the law. The equation indicates the action and reaction are universally equal without constraints for all type of bodies etc. All the laws are ought to be repeatedly confirmed by diverse and sensitive experiments and inherent characteristics of the interacting systems. But Newton's Third Law does not take any characteristic of the system in account, and is universally valid for every existing interacting system as per definition. The inherent characteristics affect the results in various laws and experiments. But Principia's third law of motion is completely independent of characteristics of bodies. The bodies can be of solid, liquid, gas or mixture of all. The law is applicable for all bodies thus can be considered for waves as well. Here those examples are discussed which were considered neither by Newton nor by following scientists quantitatively. Newton did not give any mathematical equation for the third law. Further energy is inter-convertible to various forms. Likewise Newton did not give equation for Second Law of Motion and law of gravitation. 
In Newton's time it was beginning of science in systematic way. The physical quantities, units, dimensions were not defined. Thus the terms action and reaction do not possess units and dimensions, as Newton did not define action and reaction in terms of specific physical quantities. However, in the qualitative explanation given after the definition to the law, Newton expressed action and reaction in terms of push or pull (force) and motion (velocity), in the Principia at page 20. Newton applied third law of motion to examples when body remains at rest or moves. Thus it is applicable to numerous cases, and must be confirmed quantitatively for all i.e. comparing mathematical predictions with experimental results. Newton did not write equation (1) in the Principia. Newton wrote the Principia's Second Law of Motion in proportionality form like Law of Gravitation, but The Third Law is expressed in equality form, which implies that all other factors are insignificant.

Now a day's all definitions are expressed in terms of equations. If mathematical predictions of equation is quantitatively confirmed in repeated experiments in all regions, then scientifically equations hence the definition of the law is regarded as true. Thus it is most important that definition and equation of the law is expressed in terms of physical quantity which has definite units and dimensions (may be basic or derived) i.e. Say velocity $\left(\mathrm{m} / \mathrm{s}, \mathrm{M}^{0} \mathrm{LT}^{-1}\right)$. Action and reaction neither have units nor dimensions, thus it is not logical to call them as scientific terms as give no quantitative information as required in scientific measurements. Also the word motion does not have units and dimensions and Newton himself used it as velocity. In Newton's first law the motion is regarded as velocity. Newton expressed the law of gravitation in the Principia in terms of force, mass, distance; each physical quality has definite units and dimensions.

The most of developments took place well after Newton's Principia. The dimensional analysis was initiated by Fourier? in 1822, and dimensions of force are based upon $\mathrm{F}=\mathrm{ma}$ (given by Euler in 1775), not on Principia's F $=(\mathrm{v}-\mathrm{u})$. Also, the unit of force (dyne) was first defined in 1866 , about 184 years after publication of the first edition of the Principial The $9^{\text {th }}$ Conference General des Poids et Mesures held in 1948, then adopted the name "newton" for the unit of force, in a resolution?. Thus Newton just initiated the beginning of physics by defining few terms, and did not end the same as science is expanding. Newton estimated the speed of sound in air as $280 \mathrm{~m} / \mathrm{s}$, whereas experimental value is $330 \mathrm{~m} / \mathrm{s}$. Thus Laplace corrected Newton's equation, so that exact value is obtained. Newton's law of cooling states that heat loss of a body is directly proportional to the difference in the temperatures between the body and its surroundings provided the temperature difference is small. A correction to Newton's law concerning larger temperature differentials was made in 1817 by Dulong and Petit. Thus improvements in older laws continue to accommodate as more facts as they are known.

Further, in third law of motion, the action and reaction have no units and dimensions, thus are not physical quantities. In Definition section of the Principia, Newton defined 'Quantity of mass' (mass), 'Quantity of motion' (product of mass and velocity, the momentum) at page 2, and motion (velocity) at page 10 at Scholium after Definition section. In Second law of motion in the Principia, Newton defined force as proportional to alteration in motion. But the main terms in the third law of motion, the action and reaction were not defined scientifically for quantitative equations, as they do not possess any dimensions and units.

Thus even if action and reaction are expressed in terms of mathematical quantities, the predictions are not checked quantitatively. The terms or words action and reaction are used as verb, noun, and adjective in English literature. Thus if properly analysed, then definition of Principia's third law of motion is more philosophical than scientific. Further action and reaction can be calibrated in terms of distance travelled and time taken to understand the meaning of law completely.

\section{Newton's Original Explanation of the Third Law and its Critical Analysis}

"Whatever draws or presses another is as much drawn or pressed by that other. If you press a stone with your finger, the finger is also pressed by the stone. If a horse draws a stone tied to a rope, the horse (if I may so say) will be equally drawn back towards the stone: for the distended rope, by the same endeavour to relax or unbend itself, will draw the horse as much towards the stone as it does the stone towards the horse, and will obstruct the progress of the one as much as it advances that of the other.

If a body impinges upon another and by its force 
change the motion of the other, that body also (because of the quality of, the mutual pressure) will undergo an equal change, in its own motion, towards the contrary part. The changes made by these actions are equal, not in the velocities but in the motions of bodies; that is to say, if the bodies are not hindered by any other impediments. For, because the motions are equally changed, the changes of the velocities made towards contrary parts are reciprocally proportional to the bodies. This law takes place also in attractions, as will be proved in the next scholium".

Newton gave only three qualitative examples to support the law in the Principia. In first two examples, Newton used words 'presses' or 'draws' and 'motion' in third example. Further scientists concluded that words 'presses' or 'draws' mean force in first two examples given by Newton at page 20 of the Principia. These examples are finger pushes the stone; and horse pulls the stone. The systems remain at rest and hence work done is zero. The third example is fairly general in sense when bodies move and work is done. Newton defined or meant 'motion' as velocity at pages 10 of the Principia.

If the definition of the law is expressed in terms of force and velocity (analogous to action and reaction) then the meaning of the law becomes more transparent. Clearly characteristics of system come in picture. The reason is that action and reaction are general terms, and give general meaning. However when projectile and target impinge on each other i.e. collision takes place, and then their velocities are calculated in the existing literature. The initial velocity of projectile is regarded as action and final velocity as reaction, as in case of other examples.

In the Principia, Newton did not write any equation to explain the Third Law of Motion quantitatively. For example, Newton did not measure force applied by horse on stone and force with which horse is pulled by stone. In this example horse and stone remain at rest, thus no work is done. In another example when one body (projectile) impinges (strikes) on the other (target), then bodies' move. In this case body moves and work done is non-zero. Further, there is no equation given by Newton to calculate change in motion (velocity) of projectile and target. So the illustration is qualitative, not quantitative in the Principia. Newton expressed action and reaction in third law of motion in terms of force and velocity; also applied the law for moving bodies along with bodies at rest. The velocity is related with momentum and kinetic energy. The various forms of energy are inter-convertible.
The energy is conserved in the process. Thus the third law needs to be interpreted beyond terms 'action' and 'reaction', by writing equations in terms of physical quantities. This is only the law in physics which is explained with terms which do not have units and dimensions. Thus the law needs to be further interpreted and explained, it leads to generalization of the law. In the existing physics whenever one body (projectile) impinges on the other (target), then collision is said to have taken place, and various velocities are calculated.

\subsection{Newton Represented Action and Reaction in Terms of Force and Velocity}

The basic text book for $11^{\text {th }}$ Class in Indian education system published by NCERT describes action and reaction in terms of force only. "The terms action and reaction in the third law mean nothing else but force. Using different terms for the same physical concept can sometimes be confusing The Third Law of Motion may be understood in terms of force in other parts of the world. If in the same paragraph of the Principia where Newton used words 'presses' or 'draws' and interpreted as force, Newton also used word 'motion' which Newton defined or meant as velocity. Thus, Newton also used velocity for action and reaction, as well. It is crystal clear. There is scope of confusion when we misinterpret or interpret incompletely the law. This comment itself gives way for complete and correct interpretation of the definition of Newton's Third Law of Motion. Newton too had discussed action and reaction in terms of velocity, in the same paragraph in which action and reaction were represented in terms of force in earlier part of paragraph as finger pushes the stone or horse pulls the stone. Thus action and reaction were expressed in terms of push or pull i.e. as force. In this case stone and horse remain at rest when force is applied; in this case work done is zero.

As the projectile impinges (collides) with target, it causes change in velocity (motion) of the target and experiences equal change in itself. So here action and reaction are expressed in terms of velocity. Thus applying third law of motion Newton assumed that body moves. Now this is the same perception in all types of collisions and velocities are calculated from mathematical equations in text books. Such equations were not developed in Newton's time i.e. physical phenomena were not expressed in terms of mathematical equation but 
geometrically and philosophically. In classical mechanics the mass of body remains constant. When force acts on somebody, it imparts velocity to body i.e. Causes change in velocity or try to do so. The moving body possesses velocity, momentum and energy or work, so all terms are inter-related, both conceptually and mathematically. The energy is inter-convertible from one form to other. These should not remain veiled as Newton defined law in terms of action and reaction (which has neither units nor dimensions).

Thus Newton expressed action and reaction in terms of force and velocity. Further Newton applied and elaborated the law in both cases when body moves or remains at rest. These aspects need to be critically discussed. The soul of scientific analysis is that to take all factors in account and interpret all results impartially.

In the first two examples Newton expressed action and reaction in terms of push or pull (force). In this case body remains at rest and work done is zero. It is the simplest case. In the third example Newton described impinging (colliding) bodies and expressed action and reaction in terms of velocities. Newton did not write any equation to elaborate the law. The equations for impinging or colliding bodies are written by other scientists and mathematicians when laws of conservation of energy and momentum are were defined. For one dimensional elastic collisions equations are written and elaborated in section (6). These equations have conceptual basis in the third law of motion but studied independently afterwards. Newton did not write single equation in the Principia even for all important second law of motion and law of gravitation. The exceptionally significant laws are stated as axioms or propositions and geometrically interpreted qualitatively. But now its age of precise computer programming and sophisticated robotics. Further Newton expressed the third law of motion in terms of action and reaction which did not have units and dimensions hence cannot be regarded as physical quantities. The unilateral discussion is not scientifically and logically justified. Before drawing final conclusions all aspects are required to be considered.

It is evident that action (initial velocity of projectile) and reaction (final velocity of projectile) are equal in eq.(15) i.e.

Initial speed of projectile $=-$ Final speed of projectile.

Action $\left(u_{1}\right)=-$ Reaction $\left(v_{1}\right)$
Whereas it not so other cases, in all elastic collisions the coefficient of restitution is unity. In such cases energy is converted from one form to other and total energy is conserved in the process. The eq.(15) is written under standard conditions and many factors are ignored. All factors have been considered scientifically and logically for complete understanding. Thus in collisions the properties of bodies i.e. the inherent characteristics, nature, composition, flexibility, elasticity, rigidity, magnitude, plasticity, distinctiveness of interacting bodies or mode of interactions, and other relevant factors are significant. The bodies may be of steel, wood, rubber, cloth, wool, clay, kneaded flour, chewing gum, sponge, spring, etc. The bodies can be of solid, liquid, gas or mixture of all. All these factors are significant. These factors are not taken in account by Principia's third law of motion hence the law is generalised, so that it becomes useful in explaining phenomena over wide range of problems.

\subsection{Other Factors than Action and Reaction, Not Taken in Account in Third Law}

Newton had expressed action and reaction in terms of force and velocity. Thus law can be re-stated in terms of force and velocity as action and reaction in the Principia. In terms of force,

"To every force there is always opposed an equal force; or the mutual forces of two bodies upon each other are always equal, and directed to contrary parts".

In terms of velocity, "To every velocity of a body, there is always opposed an equal velocity when it impinges on other body, or mutual velocities of two bodies upon each other are always equal and directed to contrary parts".

This law becomes more vivid, clear and transparent if discussed in this way. When two bodies collide, then force exerted by second body is not always equal to force exerted by first body. It is not guaranteed in practical examples. For example, when a rubber ball strikes the steel ball it rebounds identically to original position. Whereas when a rubber ball strikes the cotton ball it does not rebound as in case of steel ball. Newton did not mention about magnitude of target, which is very significant in such cases. This force depends upon inherent properties of body such as "the inherent characteristics, nature, composition, flexibility, elasticity, rigidity, magnitude and plasticity, distinctiveness of interacting bodies or mode of interactions. The bodies may be of steel, wood, rubber, 
cloth, wool, clay, kneaded flour, chewing gum, sponge, spring, etc. The bodies can be of solid, liquid, gas or mixture of all. The law is applicable for all bodies thus can be considered for waves as well".

Hence Third law cannot said to be universally valid in all cases as it does not take all factors in account.

For further understanding of this aspect, let us discuss how Newton defined and elaborated the third law of motion. Thus it is clear from Newton's explanation that projectile causes change in velocity of the target by its force, and suffers equal change in its own velocity in opposite direction; due to quality of mutual pressure. Newton elaborated that "quality of mutual pressure" between target and projectile causes the reaction. Obviously Newton took mutual pressure between projectile and target, as result of mutual interactions. Practically mutual interactions depend upon nature, characteristics, compositions of bodies etc. For example, mutual interactions between rubber ball and steel ball are different from mutual interactions between rubber and cotton ball. But Newton explicitly took these in mutual interactions equal for all bodies in the third law of motion. Whereas practically mutual interactions are dependent on nature, characteristics, compositions of bodies etc. These are neglected by Newton in elaborative form of Third Law of Motion. The conclusion (dependence of action and reaction on characteristics of bodies) is also evident when law is written in terms of force and velocity analogous to action and reaction. There are many experiments justify this deduction.

Further Newton did neither mention these in definition nor gave any mathematical equation which highlights or accounts for all factors via coefficient of proportionality or directly. Thus Newton's law neglects some realistic factors which can affect results, and has to be taken in account. The discoveries of Evangelista Torricelli, Von Guericke, and Robert Boyle were available at Newton's time but they are related with fluid pressure. However Newton explained the third law in terms of solid bodies only, as per definition of the law it is applicable to all bodies indiscriminately. The pressure is ratio of force and area; hence for the same force pressure can be different.

The familiar examples of Third Law of Motion is walking and swimming, person also moves possessing kinetic energy. But a person can walk neither only on rough surface not on ice. Further a person can only swim in water tank, if it has optimum depth (say $1.5 \mathrm{~m}$ ); it indicates that characteristics of medium are important. This example is different than quoted by Newton, that when a stone is pressed by finger, the stone also presses the finger. In this case finger and stone remain at rest. Newton's third law is applicable when body moves or remains at rest. There are many such examples.

Further, Newton taking other external factors in account stated that action and reaction are equal if they are not hindered by any impediments. If a projectile is pushed towards the target and it impinges (collides) on it in time $t$ after collision it rebounds. Now if some liquid, sand or other obstructing material (impediment) is splashed in path, then while coming back it would take longer time to reach at original point. Similarly strong wind (natural or artificial) can affect the results (if blows when projectile rebounds) as it also acts as impediments and hinders the motion. Thus Newton's law neglects some realistic factors which can affect results and has to be taken in account. When the law is understood in terms of action and reaction (devoid of units and dimensions), then explanation is qualitative, further Newton did not write any equation based upon the law. For comparison, now a day's all the laws are expressed in terms of equations and then predictions are experimentally checked. Newton did not write any equation; hence quantitative aspects of the law are missing.

\subsection{Calibration of Force in Terms of \\ Distances Travelled and Time Elapsed}

In general, Newton has applied or elaborated the Third Law of Motion by stating that "If a body impinges upon another and by its force change the motion of the other that body also (because of the quality of the mutual pressure) will undergo an equal change, in its own motion, towards the contrary part".

The body may impinge (strike or collide) the other if it is pushed by some force due to external influence or body may strike the other body or earth due to gravitational pull. The action and reaction can be calibrated in terms of distance travelled by body (from initial point of impact; and to then back to original point after colliding). It means relation between distance travelled and force can be established, precisely for some cases and can be extended for others. Further time (when projectile starts and strikes the target, and returns to original position) 
can be calibrated in the process like distance. Thus the action and reaction (forces exerted) can be calibrated in terms of time and distance in some cases. By measuring the distance of body the magnitude of force (work = force $\mathrm{x}$ distance) can be assessed. Such calibration can be understood for simplicity as in case of pyrometer.

[The temperature of Sun and furnaces is measured with help of pyrometer, without touching the Sun. The reason is that Pyrometer is earlier calibrated in terms of temperature. The reason is that Pyrometer is earlier calibrated in terms of temperature.

There is a definite relationship between the temperature of a body and the radiations it emits at different wavelengths. This relationship, known as the Planck's law of radiation states that the hotter the body, the brighter it is at shorter wavelengths. The Sun emits maximum at the visible or yellow wavelength and the corresponding temperature is about 6000 degree Kelvin].

Thus by calibration the understanding of Third Law of Motion is fully understood and extended in terms of time and distance, in addition to force and velocity as directly perceived by Newton. Thus applications of the Third Law of Motion are extended to its fullest extent, as the definition of the law is applicable universally. Newton expressed action and reaction in terms of force and velocity; and applied third law of motion to bodies in both cases i.e. they may be at rest or moving but quantitatively only. The comparison of original and generalized form of the third law of motion is shown in Table 1.

\subsection{Newton's Third Law of Motion is} regarded as True under Ideal Conditions Only

The law is said to be general in nature if mathematical predictions based on equation of the definition are confirmed experimentally in all possible experiments and results are quantitatively repeatable under all circumstances. These experiments may be existing at time when law is defined or discovered later on. For this first and foremost condition is that there must be concrete equation for the said law in terms of specific physical quantities (having units and dimensions). Thus predictions of mathematical equations can be experimentally checked. But Newton defined the law without mathematical equations, and explained it with three qualitative examples ignoring many others. These were not explained quantitatively. Newton expressed law in terms of force and velocity (these are associated with time and distance); also applied to bodies when they remain at rest or move. Consequently work done can be zero or non-zero. Thus there are numerous experiments where Newton's Law is applicable but not specifically applied to draw conclusions over wide range. Thus analysis of the law should not be regarded as complete. In science qualitative explanation only shows a trend of the experiments. But the law has to be fully understood with confirmation of mathematical equations in repeated experiments where Newton applied it in the Principia. The complete interpretation of the law has to be understood.

Thus Third Law may be regarded as true under ideal conditions or law cannot be regarded as confirmed under all circumstances with specific experiments in each possible case. Conceptually Newton's Third Law of Motion is applicable to numerous experiments. Newton did not give even qualitative equation to confirm the law, not to speak of quantitative one. Thus Newton's interpretation is geometrical or philosophical. The terms action and reaction have no units and dimensions, thus no equation

Table 1. Comparison of original form of Newton's law (accounts for action and reaction only) and generalized law (takes all factors in account, including action and reaction)

\begin{tabular}{|l|l|l|}
\hline Sr. No & Newton's Third Law & Generalized form of Newton's Third Law of Motion \\
\hline 1 & Action =- Reaction & $\begin{array}{l}\text { Action = -K Reaction } \\
\text { K is coefficient of proportionality }\end{array}$ \\
\hline 2 & No factors other than Action and Reaction are involved. & $\begin{array}{l}\text { "The inherent characteristics, nature, compositions, flexibility, } \\
\text { rigidity, magnitude, size, elasticity, shape, distinctiveness of } \\
\text { interacting bodies, mode of interactions, point of impact etc." } \\
\text { These factors are effective and change results. }\end{array}$ \\
\hline 3 & Neglects all other factors except action and reaction. & Takes all possible factors in account. \\
\hline 4 & Newton gave three examples, but these are qualitative. & All examples can be explained. \\
\hline 5 & $\begin{array}{l}\text { Newton expressed Action and Reaction in terms of force } \\
\text { and velocity, which has to be mathematically studied. }\end{array}$ & $\begin{array}{l}\text { In addition to force and velocity action and reaction can also } \\
\text { be calibrated in terms of time and distance. }\end{array}$ \\
\hline
\end{tabular}


can be based on these to draw quantitative conclusions. Now all equations are expressed in terms of physical quantities which have units and dimensions. Hence these are not perfect scientific terms for final understanding the concepts quantitatively. If some scientific deduction differs from this perception then law should be quantitatively confirmed in numerous experiments, where third law of motion is applicable. No scientific law is used under selective cases. So law is regarded as true under ideal cases only, not in general cases. It is further justified below with help of logical experiments.

\section{Newton did not consider the Following Experiments}

In the Principia Newton had only given three qualitative examples to justify the definition; it does not mean that now other numerous quantitative examples must be neglected or their perception does not exist. In the existing physics some examples are understood in terms of third law of motion. Also some examples are not well considered due to lack of specific mathematical equations which need more clarifications. Whereas as in case of collisions the equations are interpreted in terms of velocity in the existing literature, this discussion is application of third law of motion as Newton had initiated the same but qualitatively. The following simple experiments (along with many others) have not been considered by Newton at all while formulating and elaborating the third law of motion.

Firstly an example from the existing textbooks is considered for understanding. "Imagine a massive spring of high spring constant is being compressed with an extremely delicate device (i.e. once touched will release the spring). Now we can envision that a collision between a slowly moving particle and this device (action), would release the spring and the final kinetic energies of the massive spring and the particle will be larger than their initial one (reaction), because the elastic potential energy was converted into kinetic energy In an analogous way, instead of 'massive spring of high spring constant' as 'source of chemical energy', say an explosive can be placed, in that case also huge amount of energy would be released when a small body touches this.

Thus it is explained on the basis of inter-conversion of potential energy to kinetic energy, the other forms of energies may also be involved. The final kinetic energy may be less than initial value, the difference being ultimately converted to heat or sound or potential energy of deformation in the collision. Also final kinetic energy may exceed the initial value, as when potential energy is released in the collision. Obviously potential energy depends upon inherent nature and characteristics of body. In any case conservation of total energy holds well in all cases.

In physics, potential energy is energy possessed by a body by virtue of its position relative to others, stresses within it, electric charge, and other factors. The potential energy is of various forms (chemical, electrical, electrostatic, magnetic, nuclear etc). The various forms of energy are inter-convertible, and total energy remains constant. Similarly a spring and nail of same mass are dropped on the floor, and then spring rises upward abruptly whereas a nail of same mass and material does not rise upward.

These observations are due to reason that inherent characteristics of the projectile and target which are never taken in account in the Principia's Third Law of Motion, are correctly taken in account in generalized form of third law of motion.

Consider a rubber ball of mass $0.1 \mathrm{~kg}$. Let ball is dropped from height of 1 meter, and reaches the floor in time $t$. Then it will have weight/force $0.98 \mathrm{~N}$, the action. It will be equivalent to energy (mgh), 0.98J or work, at height of $1 \mathrm{~m}$. It must be noted that Newton had expressed action and reaction in terms of push or pull (force), motion (velocity), just after definition of the law in the Principia.

After striking the floor (action) the rubber ball rebounds (reaction) identically i.e. reach the same point ( $1 \mathrm{~m}$ above) in the same time $t$. Thus reaction is $0.98 \mathrm{~N}$ and body again possesses energy $0.98 \mathrm{~J}$. Obviously action and reaction (may be expressed in terms of force, $(\mathrm{W}=\mathrm{FS})$ are same, and Newton's law holds good.

Action $(0.98 \mathrm{~N})=-$ Reaction $(0.98 \mathrm{~N})$

In this case neither kinetic energy of ball is dissipated nor energy is added to it. So ball rebounds with same kinetic energy.

This observation is completely consistent with Newton's Third Law of Motion. It can also be understood in terms of energy i.e. ball possesses same energy before 
and after collision. The dissipation of other forms of energy is zero or negligible. Newton gave an example, that when horse pulls the stone; the stone also pulls the horse. Both horse and stone remain at rest. The reason is that Newton neither measured force applied by horse nor that by stone. In this case work done ( $\mathrm{W}=\mathrm{FS})$ is zero as body does not move at all. It must be noted there are numerous such or similar logical examples, which has to be explained with help of third law of motion.

Super elastic collisions: Consider a specially fabricated rubber (or suitable material) ball of mass $0.1 \mathrm{~kg}$. Let this ball is also dropped from height of 1 meter, on the specially fabricated floor. This ball will have action (Weight $=0.98 \mathrm{~N}$ or energy 0.98J), same as in previous case.

After striking this specially fabricated floor, the rubber ball rebound to greater height, say $2 \mathrm{~m}$ in time $\mathrm{t}$ then reaction would be double,

Reaction $=2$ Action

or Reaction $>$ Action

In this case potential energy (elastic) of the system is added to kinetic energy of ball such that it rebounds with double reaction.

Now the action and reaction are not equal in this case, now it is separate issue that why they are not equal? Thus in this case the reaction is twice the action, which is not consistent with third law. Had this specially fabricated ball (or any other typical ball) risen to height of $1 \mathrm{~m}$ only, then reaction would have been equal to action as in previous case. Hence there would have been no scope for debate on quantitative validity of the law. But in super elastic collisions the interactions of target and projectile are such that after the collision the energy is doubled. It is only possible with specially fabricated projectile and target which have special characteristics. Due to combined interactions of specially fabricated ball and floor, the ball rises to double height, as some additional energy is generated due to characteristics of the system and imparted to ball.

If all other parameters remain the same (rubber ball $0.1 \mathrm{~kg}$, height 1 meter) as in first case but now ball falls on the sand or soft soil. Now we find that the rubber ball rebounds to 0.01 meter (or less) from sand or soil; as in previous cases (from ordinary floor, specially fabricated ball and specially floor) it rebounded to $1 \mathrm{~m}$ and $2 \mathrm{~m}$. The action $(0.98 \mathrm{~N}$ or $0.98 \mathrm{~J})$ is same in all the three cases but reactions are different, as characteristics are also different. But in literature mainly first case is discussed as for suitability of conclusion. The inherent characteristics of floor and sand are entirely different causing different interactions between projectiles and targets. Thus the rubber ball of same mass rebounded to the lesser height means possesses lesser reaction (force) than previous case. Hence

Reaction $=0.01$ Action

or Reaction $<$ Action

In this case almost whole the kinetic energy of ball is converted to potential energy of sponge depending upon its characteristics and comparatively negligible energy is imparted to ball.

It is undoubtedly clear that the reaction depends upon the inherent characteristics of system, which are not taken in account by the Principia's Third Law of Motion.

Further, if a typical sponge ball of mass $0.1 \mathrm{~kg}$ $(\mathrm{W}=0.98 \mathrm{~N}$, energy $=0.98 \mathrm{~J}$ or the action) is dropped from the height $1 \mathrm{~m}$ on the soft surface. Then it does not rebound at all. Thus

Action $=0.98 \mathrm{~N}$

Reaction $=0$

Again action and reaction are not equal. As sponge ball stays on the soft surface ( $h=0$ ), thus kinetic energy is zero. It is due to inherent nature, characteristics, compositions etc. As due to these factors the interactions of projectile and target are different. Action and reaction would have been equal if the sponge ball would have risen to original height of $1 \mathrm{~m}$ in time $t$. The reaction is also zero in NASA's EM Drive experiment published in peer review journal.]

Newton simply maintained universal equality between action and reaction, but he did not mention about the magnitude and characteristics of target. It can be understood in practical and logical examples. If the golden ball of mass $0.1 \mathrm{~kg}$ ( $\mathrm{W}=0.98 \mathrm{~N}$ or Energy $=0.98 \mathrm{~J})$ is dropped on the stretched paper, then it pierces through it. The reason is that the paper is unable to offer even fraction of reaction compared to action due to characteristics of target. It justifies that action and reaction are not universally equal. Thus due to this paper cannot stop ball and later tears it and then falls down. When the same ball 
falls on the floor, gets sufficient reaction. The similar is the reason a bullet pierces through the wall, tree or human body. Similarly for swimming a person needs to have tank having minimum depth.

Thus the Third Law of Motion is true under special or ideal or most suitable conditions only. Had Newton thought about these and similar other experiments he would have given the Third Law of Motion in different way i.e. in generalized form.

Thus it is evident from various discussed examples, that different reactions for same mass (same action) are different due to inherent characteristics, nature, compositions, flexibility, rigidity etc. of projectiles and targets. The Principia's third law neglects all these factors. Hence third law has been generalized or extended so that the elusive factors are taken in account.

NASA's Reactionless drive. It is drastic improvement of perception of English Satellite Engineer ${ }^{10}$, that reaction less space craft's are possible. This EM (Electromagnetic) Drive engine appeared to violate the third Law of Motion which vindicated previously published but unreviewed results of similar devices. Harold White lead team concluded in the paper; "Thrust data from forward, reverse, and null suggests that the system is consistently performing with a thrust to power ratio of $1.2 \pm 0.1$ millinewtons per kilowatt".

That's not an insignificant amount - to put it into perspective, the super-powerful Hall thruster generates force of 60 millinewtons per kilowatt, an order of magnitude more than the EM Drive. Hall Thruster is based on exhaust of material. Thus the results from NASA's reactionless drive are the significant, but must be repeated for final confirmation. The Cannae Drive (plans to eventually launch a cubesat satellite) and other drive developed by Chinese scientist Yang work on the same idea. More experiments are being conducted by different scientists in different regions.

- Let a rocket weighing equal to $3,000 \mathrm{~kg}$ is moving with speed $2,00 \mathrm{~m} / \mathrm{s}$ i.e. momentum (forward) $6 \times 10^{5} \mathrm{~kg} \mathrm{~m} / \mathrm{s}$. Third law implies that the backward momentum of exhaust (gases, smoke etc.) must be equal to $6 \times 10^{5} \mathrm{~kg} \mathrm{~m} / \mathrm{s}$. But such measurements have never been reported, as it is impossible to measure mass of exhaust at any instant. Without precise experiments in this case Third Law must be regarded as qualitatively true only. When a bird flies in air, it presses the air with wings as action, and as reaction the bird moves forward. Thus it is a qualitative example of application of third law of motion.

In NASA's recent EM drive experiment published after peer review, there is no exhaust. The toys aero planes fly with electronic batteries do not emit any exhaust required for validity of third law. As exhaust is necessary for rocket, then it would have also been requirement for motion of passenger planes, helicopters, bus, truck, train, ship, moving electronic equipments etc. These various machines or devices move under entirely different conditions, but the basic laws describing their motion must be the same. The repetitions can be done in vacuum to eliminate resistance due to air. The trials can be made by reducing the resistive forces to bare minimum of the system as well. Newton himself mentioned that bodies should not be hindered by any other impediments, which is also basis of Galileo's Law of inertia and Newton's first law utilizes the same basis. NASA's EM drive experiments too have been conducted in vacuum purposely. The scientific results cannot be drawn from unilateral analysis.

The action and reaction (do not possess units and dimensions) are indirectly understood in terms of force and velocity, and can be calibrated in terms of time and distance. Had Newton expressed the law in terms of force and velocity (have units and dimensions) directly then understanding would have been much easier.

- In statistical mechanics there is a variety of situations in which Newton's third law (action = reaction) is violated. Generally, the action-reaction symmetry can be broken for mesoscopic particles, when their effective interactions are mediated by a nonequilibrium environment. This violation and the resulting breaking of the force symmetry can occur when there is a relative motion of interacting particles versus an interface. The issue is discussed in details the paper Statistical Mechanics where Newton's Third Law is broken ${ }^{11}$. Thus such experimental discussions support generalized form of Newton's Third Law of Motion, which implies that action and reaction may or may not be equal.

Thus as investigations continue, many new facts about the law are emerging and final confirmations can be drawn with repeated experiments. The action and reaction are shown for various bodies having different characteristics on the variety of surfaces are shown in Table 2. 
Table 2. Comparison of action and reaction when body of mass $(0.1 \mathrm{~kg}$ or $0.98 \mathrm{~N}$, Action) of different characteristics striking at different surfaces when dropped from the same height. The action is same for all bodies $(0.1 \mathrm{~kg}$ is $0.98 \mathrm{~N})$ but reactions may be different

\begin{tabular}{|l|l|l|l|l|l|}
\hline $\begin{array}{l}\text { Sr. } \\
\text { No. }\end{array}$ & Projectile Ball (0.1kg) & Striking surface & $\begin{array}{l}\text { Action } \\
\text { (body falls) }\end{array}$ & $\begin{array}{l}\text { Reaction } \\
\text { (body rebounds) }\end{array}$ & Deduction \\
\hline 1 & Rubber & Floor & $1 \mathrm{~m}$ & $1 \mathrm{~m}$ & Action $=-$ Reaction \\
\hline 2 & Rubber & soil/sand & $1 \mathrm{~m}$ & $0.01 \mathrm{~m}$ & Reaction $=-0.01$ Action \\
\hline 3 & $\begin{array}{l}\text { Specially fabricated } \\
\text { rubber ball }\end{array}$ & $\begin{array}{l}\text { Specially fabricated } \\
\text { surface }\end{array}$ & $1 \mathrm{~m}$ & $2 \mathrm{~m}$ & Reaction $=-2$ Action \\
\hline 4 & Sponge & Soft surface & $1 \mathrm{~m}$ & 0 & Reaction $=0$ \\
\hline 6 & NASA's EM drive & Experimental set up & Microwaves Action $>0$ & 0 & Reaction less device \\
\hline 5 & Golden ball & Paper & $1 \mathrm{~m}$ & 0 & Reaction? (paper torn) \\
\hline
\end{tabular}

\section{The Various Projectiles Strike on the Various Targets}

The affect of characteristics of the system on the action and reaction law can be understood with help of other simple and logical examples. These experiments are in the realm of applicability of the third law of motion; hence it is absolutely necessary to discuss them over wide range. Due to any reason (such as Newton gave just three qualitative examples to explain the law, Newton did not give any mathematical equation, Newton stated law in terms of action and reaction and reaction which did not have units and dimensions) the application and explanation of the law should not be limited to certain experiments only. Consider a gun or injecting/firing device is specially fabricated for throwing various bodies with known force on the target (concrete wall, say). Let the gun is placed at 5 meters away from the wall and ejects or fires rubber ball.

(i) The rubber ball is thrown to the wall after firing the gun and reaches the target at $5 \mathrm{~m}$ in time t. Let the force applied on the ball be $\mathrm{F}_{\text {action }}$. The ball strikes the wall and rebounds to original position travelling distance $5 \mathrm{~m}$ in time $\mathrm{t}$. Thus, the ball rebounds or retraces its path identically. The $\mathrm{F}_{\text {reaction }}$ and $\mathrm{F}_{\text {action }}$ are same.

In this case neither original kinetic energy of ball is dissipated nor additional energy is added to it, hence ball rebounds with original kinetic energy.

$\mathrm{F}_{\text {action }}=-\mathrm{F}_{\text {reaction }}$

Thus Newton's Third Law is obeyed. As the force and distance travelled are the same, thus initial energy is equal to final energy. Thus clear meaning of the law is under veil as Newton expressed it in terms of action and reaction, and some phenomena are understood qualitatively only. The Principia's interpretation also limits the vast applications of the third law of motion. The major reason for this is that in Newton's time units and dimensions and mathematical equations (algebraic or analytical) were not discovered, so Newton used philosophical and geometrical methods to interpret the phenomena.

(ii) Let a rubber ball is specially fabricated using different material. The rubber ball is thrown on the wall after firing the gun and reaches the target at $5 \mathrm{~m}$ in time $\mathrm{t}$. Thus, action is $\mathrm{F}_{\text {action }}$. After striking, the ball rebounds to original position in time $t / 2 \mathrm{~s}$. Thus in this case reaction will be $\mathrm{F}_{\text {reaction }}$ or $2 \mathrm{~F}_{\text {action }}$. Thus

$\mathrm{F}_{\text {reaction }}=2 \mathrm{~F}_{\text {action }}$ or $\mathrm{F}_{\text {action }} \neq \mathrm{F}_{\text {reaction }}$

In this case potential energy (elastic) of system is converted to kinetic energy of ball and added to it such that it rebounds with double reaction.

As discussed previously, the inherent nature, characteristics, composition etc. of the projectile and target are responsible for different action and reaction. These experiments are different from Newton's perception that finger presses stone and stone presses the finger. Both remain at rest, hence work done is zero.

(iii) The characteristics and magnitude of target are significant, but are not discussed by Newton in the third law at all. Now consider that the concrete wall is replaced by cardboard wall. The rubber ball is thrown at the wall after firing the gun and reaches the target at $5 \mathrm{~m}$ in time t. Like previous cases, in this case also third law is applicable. Let the force applied on the wall be $\mathrm{F}_{\text {action }}$. Now as the rubber ball impinges (strikes) at the 
cardboard wall, it breaks and rubber ball crosses across the broken cardboard wall. The cardboard does not offer any reaction (as wall does to ball in previous cases) or offers insufficient reaction or loses own identity during collision. Thus the characteristics of projectile and target are very important in such cases; Newton's law does not take them in account. $\mathrm{F}_{\text {action }}$ on the rubber ball is same as in case of previous cases. Again the characteristics of projectile and target play significant role. The ball does not rebound, moving through cardboard wall hence,

$\mathrm{F}_{\text {reaction }}=0$

$\mathrm{F}_{\text {action }} \neq \mathrm{F}_{\text {reaction }}$

Thus third law of motion is not obeyed. Newton did not mention about such experiments in the Principia.

(vi) Consider a chewing gum ball of the same mass (as in above two cases). The chewing gum is thrown on the wall after firing the gun and reaches the target at $5 \mathrm{~m}$ in time t. After striking the wall the chewing gum sticks to wall and does not rebound. Thus

$\mathrm{F}_{\text {reaction }}=0$

Hence in this case Newton's third law is not obeyed.

$\mathrm{F}_{\text {action }} \neq \mathrm{F}_{\text {reaction }}$

Thus characteristics of bodies are entirely significant in imparting the reaction to projectile. The definition of Newton's third law of motion does not take these aspects in account but the generalized form of third law of motion accounts for all such factors.

This is again due to different inherent characteristics of projectile and target. Thus whole the kinetic energy is converted to potential energy. Or the chewing ball develops the strong binding of adhesion on the wall due to chemical energy.

(v) Further Newton's law is completely silent about electrical, magnetic and similar other interactions between the various bodies, these are exception in some cases.

Consider two magnets A and B of equal and significant pole strengths. Let both the magnets (Newton's third law of motion is applicable for all bodies) are placed on the smooth table at distance of $1 / 2 \mathrm{~m}$ (say).

(a) Let the north pole of magnet $B$ is pushed with force
Faction towards South Pole of magnet a (stationary). As the opposite poles attract each other, so both the magnets are stuck to each other. Thus magnet B does not re-trace its path, as according to Newton to every action there must be reaction (motion in opposite direction). But in this case reaction is zero i.e.

Thus whole kinetic energy of chewing gum ball is converted to potential energy (chemical), thus force of adhesion between ball and wall becomes exceptionally high. Thus no kinetic energy is imparted to chewing gum ball; hence it sticks to ball and does not rebound.

$$
\mathrm{F}_{\text {reaction }}=0 \quad \text { and } \quad \mathrm{F}_{\text {action }} \neq \mathrm{F}_{\text {reaction }}
$$

The potential energy (magnetic) is strongly developed as attractive energy between opposite poles of magnet due to inherent characteristics and interactions. But this type of energy is not developed in two similar iron bars.

This aspect is not taken in account in third law of motion.

(b) Let the north pole of magnet B is pushed with force $\mathrm{F}_{\text {action }}$ towards North Pole of magnet $\mathrm{A}$. The similar poles of magnets repel each other, and magnet may be repelled with more velocity than it is pushed. It depends upon the strength of magnets. When magnets come in the magnetic field of each other, they repel each other and move away. There is definite reaction without physical contact.

(c) If North Pole of magnet A is gently placed near North Pole of magnet B without pushing the magnet. Thus analogous to previous cases, in this case $\mathrm{F}_{\text {action }}=0$. The magnet A moves away from magnet $\mathrm{B}$ which implies that $\mathrm{F}_{\text {reaction }}$ is non-zero. Thus characteristics of bodies (law is applicable for all bodies universally as per definition where action and reaction are involved) play significant role, and all aspects must be taken in account before drawing final conclusions. Newton had reported some general experiments with magnets, but did not apply action and reaction law to attraction and repulsion law to magnets, which is equally applicable like striking two bodies (iron bars).

For comprehensive understanding such experiments can be conducted in space under zero or reduced gravity conditions. All these experiments relating to third law of motion can also conducted on the earth by reducing the resistive forces. The comparison of actions and reactions are shown for various bodies in the Table 3 . 
Table 3. Comparison of action and reaction, for various projectiles colliding various targets

\begin{tabular}{|c|c|c|c|c|c|}
\hline Sr. No. & Projectile & Target & Action & Reaction & Third Law of Motion \\
\hline 1 & Rubber ball & Concrete wall & $\mathrm{F}_{\text {action }}$ & $\mathrm{F}_{\text {action }}$ & Action $=$ Reaction \\
\hline 2 & Rubber ball (special) & Concrete wall (specially fabricated) & $\mathrm{F}_{\text {action }}$ & $2 \mathrm{~F}_{\text {action }}$ & $\mathrm{F}_{\text {action }} \neq \mathrm{F}_{\text {reaction }}$ \\
\hline 3 & Rubber wall & Cardboard wall & $\mathrm{F}_{\text {action }}$ & 0 (wall breaks $)$ & $\mathrm{F}_{\text {action }} \neq \mathrm{F}_{\text {reaction }}$ \\
\hline 4 & Chewing gum ball & Concrete wall & $\mathrm{F}_{\text {action }}$ & 0 ( ball sticks) & $\mathrm{F}_{\text {action }} \neq \mathrm{F}_{\text {reaction }}$ \\
\hline 5 & Magnet B (pushed) & $\begin{array}{l}\text { Magnet A, opposite pole (station- } \\
\text { ary) }\end{array}$ & $\begin{array}{l}\text { Push } \\
\left(\mathrm{F}_{\text {action }}\right)\end{array}$ & $\begin{array}{l}\text { Attraction } \\
\left(\mathrm{F}_{\text {reaction }}\right)\end{array}$ & $\mathrm{F}_{\text {reaction }}>\mathrm{F}_{\text {action }}$ \\
\hline 6 & $\begin{array}{l}\text { Magnet A, north pole } \\
\text { (stationary ) }\end{array}$ & Magnet B, north pole (stationary) & \begin{tabular}{|l} 
Push \\
$\left(\mathrm{F}_{\text {action }}\right)$ \\
zero
\end{tabular} & $\begin{array}{l}\text { Repulsion } \\
\left(\mathrm{F}_{\text {reaction }}\right) \\
\text { non zero }\end{array}$ & $\begin{array}{l}\mathrm{F}_{\text {action }}=0 \\
\mathrm{~F}_{\text {reaction }} \text { is Non-zero }\end{array}$ \\
\hline
\end{tabular}

\section{The reasons for inconsistent results}

Newton did not give any equation to measure or calculate the magnitudes of action and reaction. The action and reaction do not possess units and dimensions. The mathematical equations of the law must be expressed in terms of physical quantities which have units and dimensions. Only then law is checked quantitatively in sensitive experiments. Newton's explanation is only qualitative. Thus Newton provided conceptual, thematic and philosophical explanation of the law, not quantitative which is understandable for scientific developments in $17^{\text {th }}$ century. With dawn or progress of industrial revolution between 1760 to 1840 , the quantitative measurements were absolute requirements of the involved quantities. Newton's law is unconditionally applicable to all interacting bodies, action and reaction are always equal and opposite for all interacting bodies. In the Table 2, Table 3 and Table 4, the first example in each case is consistent with Principia's Third Law of Motion. But it is not prudent and scientific that only highlighting few examples, the law is regarded as universally true, like its definition. One may argue that ball bounces to different heights due to reason they have different inherent properties. It is true but the Principia's third law of motion does not take in account the inherent characteristics, nature, composition, flexibility etc. in account for projectile and target.

The Principia's law does not take in account the inherent characteristics, nature, composition, flexibility, rigidity, magnitude, size, distinctiveness of interacting bodies etc. There is no term, which accounts for the above significant factors. The bodies may be composed of steel, wood, rubber, cloth, wool, sponge, spring, elastic, plastic, typical plastic, porous material, mud or kneaded flour or chewing gum, especially fabricated etc. The bodies may be solids, liquids, gases, or mixture of all. These factors play important role in action and reaction phenomena. In the Principia's third law Newton blatantly neglected these factors as they do not exist. These are very significant factors affecting the results and are taken in account via a coefficient of proportionality (K) in the generalized law i.e. equation (31). The value of $\mathrm{K}$ is determined experimentally. Due to these factors different bodies experience different reaction for same action. So Newton did not mention these factors at all and summed up the basic law just giving three qualitative examples. Just possible that Edmund Halley (sponsor) wanted the manuscript as soon as possible. Also other contemporary scientists like Robert Hook and Leibniz were raising priority issues, which were diplomatically dealt by sponsor Halley. Thus Newton might have completed the manuscript quickly, and other scientists learnt about the contents when it was published. In the preface of the Principia, Newton did not mention name of any scientist who had read the manuscript. In Newton's time phenomena were expressed geometrically and philosophically, so Newton did. Thus in the Principia, no linear equation or analytical equation were used to interpret the phenomena quantitatively. Further action and reaction are used in English language as verb, noun, and adjective. In science units and dimensions are absolutely important to interpret the phenomena. These are not physical quantities hence cannot be used to represent the equation to draw conclusions quantitatively. In Newton's time units and dimensions were not discovered, it is hard reality. Due to this reason above elusive factors may have not been taken in account in third law of motion. Hence the law is generalized. 
Table 4. The theoretical comparison of action and reaction (initial and final velocities of projectile) under ideal conditions, in elastic collisions (coefficient of restitution, e=1). The Principia's Third Law is obeyed in first case only

\begin{tabular}{|l|l|l|l|l|l|l|}
\hline $\begin{array}{l}\text { Sr. } \\
\text { No }\end{array}$ & $\begin{array}{l}\text { Projectile } \\
(\text { mass })\end{array}$ & Target (mass) & $\begin{array}{l}\text { Initial velocity } \\
(\text { action})\end{array}$ & $\begin{array}{l}\text { Final velocity } \\
(\text { reaction })\end{array}$ & Action = -Reaction & $\begin{array}{l}\text { Third Law } \\
\text { Obeyed }\end{array}$ \\
\hline 1 & $\mathrm{M}_{1}$ & $\mathrm{M}_{2}>>\mathrm{M}_{1}$ & $\mathrm{u}_{1}$ & $-\mathrm{u}_{1}$ & Action =-Reaction & Yes \\
\hline 2 & $\mathrm{M}_{1}$ & $\mathrm{M}_{2}=1000 \mathrm{M}_{1}$ & $\mathrm{u}_{1}$ & $-0.998002 \mathrm{u}_{1}$ & Action $=-1.002$ Reaction & No \\
\hline 3 & $\mathrm{M}_{1}$ & $\begin{array}{l}\mathrm{M}_{2}<<\mathrm{M}_{1} \\
\left(\mathrm{u}_{2}=0\right)\end{array}$ & $\mathrm{u}_{1}$ & $+\mathrm{u}_{1}$ & Reaction=0, No rebound & No \\
\hline 4 & $\mathrm{M}_{1}$ & $\mathrm{M}_{1}=\mathrm{M}_{2}$ & $\mathrm{u}_{1}$ & $\mathrm{v}_{1}=0$ & Reaction $=0$, projectile at rest & No \\
\hline 5 & $\mathrm{M}_{1}$ & $\mathrm{M}_{2}=1.01 \mathrm{M}_{1}$ & $\mathrm{u}_{1}$ & $-0.004975 \mathrm{u}_{1}$ & Action $=201$ Reaction & No \\
\hline 6 & $\mathrm{M}_{1}$ & $\mathrm{M}_{1}=\mathrm{M}_{2}$ & $\mathrm{u}_{1}$ & $\mathrm{u}_{2}$ & Reaction=0, No rebound & No \\
\hline
\end{tabular}

\section{The Various Types of Collisions and the Third Law of Motion}

In the third example of explanation of third law of motion in the Principia at page 20 , elaborated the impinging or colliding bodies. But Newton did not give any equation for the same due conceptual understanding at that time. This aspect is completely studied in terms of various collisions by following scientists in concrete way when the law of conservation of kinetic energy and momentum were defined. However, Newton had laid foundations of collisions, which are investigated quantitatively here. The equations for projectile and target were written before and after collision in terms of velocity. The initial velocity of target is termed as action and final velocity as reaction. The equations are well-known for various types of collisions but for simplicity here one dimensional elastic collisions are considered. Now it becomes amply clear that Newton's third law does not account for the significant factors e.g. inherent characteristics, nature, compositions, flexibility, rigidity, magnitude, size, elasticity, shape, distinctiveness of interacting bodies, mode of interactions, point of impact etc. The law is universally applicable for all bodies e.g. bodies may be of steel, wood, rubber, cloth, wool, sponge, spring, typical plastic, porous material, air / fluid filled artifact, mud or kneaded flour or chewing gum specifically fabricated material etc. All these factors and characteristics of the system must be considered for proper understanding. Thus law is generalized that all factors are taken in account.

Newton expressed action and reaction in third law of motion in terms of both 'force 'and 'velocity' at page 20 of the Principia. Newton's explanation implies that law is applicable for both situations when body is at rest (in this case work, $\mathrm{W}=\mathrm{FS}$ is zero) and moves. Thus scientifically it prudent that law must be studied critically and quantitatively for the fullest understanding of the law. In Newton's time laws were explained philosophically and geometrically not mathematically (algebraically and analytically). In third example Newton quoted in the Principia. "If a body impinges upon another and by its force change the motion of the other, that body also will undergo an equal change, in its own motion, towards the contrary part".

This Newton himself applied his third law of motion in the impinging or colliding bodies. The explanation is equally applicable even if target is at rest or moving, there is no constraint. This application of the third law of motion (impinging or colliding of one body on the other) finds many applications in various branches of physics. The target may be at rest or moving without any constraint. Newton did not characterize projectile and target in third example, whereas in first two examples Newton mentioned finger presses stone; and horse pulls stone. In the third example Newton did not mention any body specifically. However law is applicable to all bodies universally. In highly technological physics nuclear reactors heavy water is used as moderator (to slow down fast moving neutrons to thermal neutrons), is also based upon such mathematical calculations (however under ideal conditions as practically situation is entirely different).

Consider one-dimensional elastic collisions ${ }^{12}$. When one body impinges or collides other. Furthermore, in collisions comparative size and point of impact, of target and projectile, roughness of surfaces and resistive forces play significant roles. The consideration of heat, light, sound energy etc. May have significant impact in 
some cases. But such factors are not mentioned except velocities and masses $\left(M_{1}, M_{2}\right.$ in general sense) taken in equations. Also Newton did not mention about these when stated that one body impinges (collides) on the other (there are numerous bodies with entirely different characteristics). In collisions the mathematical equations indicate law is true under ideal or special conditions only, not in general . Regarding collisions there are well defined equations in the existing physics. Whereas in sub-sections (3-4) equations are written mainly in terms of action and reaction. The uniformity in explanation can be maintained for scientific understanding.

The definition is universally applicable irrespective of characteristics of the projectile and target. Collisions in which both momentum and kinetic energy of the system are conserved are called elastic collisions. These equations (10-30) are based upon conditions of applicability of elastic collisions (conservation of momentum, conservation of kinetic energy) and coefficient of restitution or coefficient of resilience is unity $(e=1)$. Then in view of Third Law of Motion action (initial velocity of projectile) and reaction (final velocity of projectile) can be compared to draw conclusions. Consider projectile and target of masses $M_{1}$ and $M_{2}$ moving along the same straight line with speeds $\mathrm{u}_{1}$ and $\mathrm{u}_{2}$ respectively. The bodies will collide only if $\mathrm{u}_{1}>$ $\mathrm{u}_{2}$. The final speeds of projectile and target are $\mathrm{v}_{1}$ and $\mathrm{v}_{2}$,

$\mathrm{v}_{1}=\frac{\left(M_{1}-M_{2}\right) u_{1}+2 M_{2} u_{2}}{\left(M_{1}+M_{2}\right)}$

$\mathrm{v}_{2}=\frac{\left(M_{2}-M_{1}\right) u_{2}+2 M_{1} u_{1}}{M_{1}+M_{2}}$

If the target is at rest $\left(\mathrm{u}_{2}=0\right)$, then Eqs. (10-11) become

$\mathrm{v}_{1}=\frac{\left(M_{1}-M_{2}\right) u_{1}}{\left(M_{1}+M_{2}\right)}$

$\mathrm{v}_{2}=\frac{2 M_{1} u_{1}}{M_{1}+M_{2}}$

The initial velocity $u_{1}$ of the projectile is regarded as action, and its final velocity $\mathrm{v}_{1}$ as reaction. The various sub-cases are discussed below.

When $M_{2}>>M_{1}$ i.e. target is very massive compared to the projectile. The target $\mathrm{M} 2$ remains at rest $\mathrm{v}_{2}=0$. For example, when projectile (ball) collide with huge target (wall). Thus

$$
\mathrm{M}_{1}-\mathrm{M}_{2}=-\mathrm{M}_{2}, \quad \mathrm{M}_{2}+\mathrm{M}_{1}=\mathrm{M}_{2}
$$

In this case the final speeds of the projectile and target can be calculated from equations (12-13).

$\mathrm{v}_{1}($ reaction $)=\frac{-M_{2} u_{1}}{M_{2}}=-\mathrm{u}_{1}$ (action)

Final speed of projectile $=-$ Initial speed projectile.

Action $\left(\mathrm{u}_{1}\right)=-$ Reaction $\left(\mathrm{v}_{1}\right)$

The velocity of target, $\mathrm{v}_{2}=0$

Thus projectile (ball) rebounds with original velocity and target (wall) remains at rest, the third law is obeyed. The negative sign in $\mathrm{v}_{1}$ indicates that direction of projectile reverses after collision.

$\mathrm{e}=\frac{v_{2--} v_{1}}{u_{1}-u_{2}}=\frac{u_{1}}{u_{1}}=1$

As the coefficient of restitution is unity, the collision is elastic.

Ideal calculations only: However these are ideal mathematical calculations as we have not considered actual experimental characteristics at all i.e. magnitude, shapes, sizes of projectile and target, point of impact, resistive force, compositions etc. The rest of calculations are based on similar perceptions. The composition of various bodies may be different同 e.g. body B can be of cloth and body A of wood, body B can be water filled ball and body A of aluminum, body B may be an air filled football and body A of gold or various types of bodies can also be considered. The definition of the third law of motion is applicable for all bodies universally. The various experiments must be conducted before drawing final conclusions. Experimentally situation is different than ideal mathematical considerations.

The effect of such bodies are neither discussed in third law of motion nor in mathematical equations. The equations are written and interpreted under standard or ideal conditions in the existing literature. The generalized form of the third law of motion accounts for all such cases.

Mass of target is 1000 times larger than that of projectile. If we consider that the target is 1000 times more massive than the projectile i.e. $M_{2}=1000 M_{1}, M_{1}+M_{2}$ $=1001 \mathrm{M}_{2}, \mathrm{M}_{1}-\mathrm{M}_{2}=999 \mathrm{M}_{2}$ then equation (9) becomes

$\mathrm{v}_{1}=-0.998001998 \mathrm{u}_{1}$ 
Initial velocity of projectile or action $=1.002002$ final velocity of target or reaction

Action $\left(\mathrm{u}_{1}\right)=-1.002002$ Reaction $\left(\mathrm{v}_{1}\right)$

Thus Newton's third law of motion is not justified in this case. The collision is elastic as

$\mathrm{e}=\frac{v_{2--} v_{1}}{u_{1}-u_{2}}=\left[\frac{2 M_{1} u_{1}}{1001 M_{1}}-\frac{-999 M_{1} u_{1}}{1001 M_{1}}\right] /\left[\mathrm{u}_{1}-0\right]=1$

(iii) Let us consider the case when the target is very- 2 small compared to projectile. $\left(\mathrm{M}_{2}<<\mathrm{M}_{1}\right.$ or even mass of target may be negligible compared to projectile). $M_{2}+M_{1}$ $=\mathrm{M}_{2}$. The various velocities can be calculated. Now from equations (12-13).

$\mathrm{v}_{1}=\frac{M_{1} u_{1}}{M}=\mathrm{u}_{1}$

Reaction $=$ Action

Thus the projectile does not rebound at all and keep on moving with original velocity, which is contrary to original form of third law of motion i.e. 'to every action there is opposite and equal reaction. The projectile keeps on moving with same velocity.

It implies that inherent characteristics, nature, magnitude, shape, size, point of impact of projectile and target play significant roles in experiments. The body is moving with velocity $\mathrm{u}_{1}$ as in earlier case. These are not taken in account by original form of law; hence Principia's law is generalized. Thus for every collision reaction is not possible.

$\mathrm{v}_{2}=\frac{2 M_{1} u_{1}}{M_{1}}=2 u_{1}$

The final velocity of target $=2$ initial velocity of projectile.

This situation can be theoretically understood by visualizing a ball hanging in air in train track. The moving train (projectile, $M_{1}$ ) hits the ball (target, $M_{2}$ ); the ball moves with double velocity of train and train keep on moving with original velocity. Anyhow this prediction needs to be confirmed under controlled and measurable conditions in the laboratory, only then final conclusions can be drawn. Obviously it will highlight the importance of characteristics of bodies regarding experimental confirmation the law. As already mentioned Newton's law does not take the characteristics of the bodies in account, so such experiments are very significant. Further,

$\mathrm{e}=\frac{v_{2--} v_{1}}{u_{1}-u_{2}}=\frac{2 u_{1}-u_{1}}{u_{1}}=1$

(vi) Let us consider the target is slightly heavier than projectile i.e. $\mathrm{M}_{2}=1.01 \mathrm{M}_{1}$.

Thus, $\quad \mathrm{M}_{1}-\mathrm{M}_{2}=\mathrm{M}_{1}-1.01 \mathrm{M}_{1}=-0.01 \mathrm{M}_{1}, \quad \mathrm{M}_{1}+\mathrm{M}_{2}=$ $\mathrm{M}_{1}+1.01 \mathrm{M}_{1}=1.01 \mathrm{M}_{1}$

In this case the final velocities of projectile and target are can be calculated from equations (12-13)

$\mathrm{v}_{1}=\frac{\left(M_{1}-1.01 M_{1}\right) u_{1}}{\left(M_{1}+1.01 M_{1}\right)}=\frac{-0.01 M_{1} u_{1}}{2.01 M_{1}}=-0.004975 \mathrm{u}_{1}$

Final velocity of projectile $=-0.004975$ Initial velocity of projectile.

Action $=-201.005$ Reaction

The negative sign in $\mathrm{v}_{1}$ indicates that direction of projectile after collision reverses. But magnitudes of action and reaction are different, thus third law is not obeyed.

This case is precisely used in the nuclear reactor to reduces the speed of fast moving neutrons $(2 \mathrm{MeV}$, $\left.1,95 \times 10^{4} \mathrm{~km} / \mathrm{s}\right)$ to thermal energy $(0.025 \mathrm{MeV}, 2.2 \mathrm{~km} / \mathrm{s})$ but under ideal conditions. The fast moving neutron $\left(\mathrm{m}_{\mathrm{n}}=\right.$ $1.00866 \mathrm{u}$ ) are made to hit the proton $(1.007276 \mathrm{u})$ in heavy water. Thus velocity of neutron can be calculated when it strikes with proton, say with individual proton (assumed) like two marbles, which is only ideal conception, can be calculated. It turns out to be nearly $13 \mathrm{~km} / \mathrm{s}$ i.e. about 5.9 times the initial speed. But practically other factors are also there which are neglected, so the speed of neutron may be practically reduced to thermal neutrons which cause fission. Purposely other moderators are also used.

$\mathrm{v}_{2}=\frac{2 M_{1} u_{1}}{\left(M_{1}+1.01 M_{1}\right)}=\frac{2 M_{1} u_{1}}{2.01 M_{1}}=0.995024 \mathrm{u}_{1}$

The coefficient of restitution in this case is also unity i.e.

$\mathrm{e}=\frac{v_{2--} v_{1}}{u_{1}-u_{2}}=\left[\frac{2 M_{1} u_{1}}{2.01 M_{1}}+\frac{-0.01 M_{1} u_{1}}{2.01 M_{1}}\right] /\left[\mathrm{u}_{1}-0\right]=1$ 
(v) Let us consider the case when masses of projectile and target are equal i.e. $\mathrm{M}_{1}=\mathrm{M}_{2}=\mathrm{M}$.

Now consider the case when target is at rest $\left(\mathrm{u}_{2}=0\right)$, then we get,

$\mathrm{v}_{1}=\frac{0}{M+M}=0$

The projectile comes to rest after collision i.e. reaction $=0$

$\mathrm{v}_{2}=\frac{2 M u_{1}}{2 M}=\mathrm{u}_{1}$

Final velocity of target $=$ Initial velocity of projectile

Experimentally it can be easily observed if one marble properly hits other in typical collision; then one marble (projectile) comes to rest and target starts moving with velocity of projectile. The children practically observe this situation while playing with marbles in streets in few cases. In this case direction of the projectile not reversed which is condition for validity of Third Law of Motion.

(vi) Let us consider the case when masses of projectile and target are equal i.e. $\mathrm{M}_{1}=\mathrm{M}_{2}=\mathrm{M}$. The various velocities can be calculated $\left(\mathrm{u}_{2}>0\right)$. Now from equations $(10-11)$ we get

$\mathrm{v}_{1}=\frac{\left(M_{1}-M_{2}\right) u_{1}+2 M_{2} u_{2}}{\left(M_{1}+M_{2}\right)}=\frac{0+2 M u_{2}}{M+M}$

$\mathrm{v}_{1}($ reaction $)=\mathrm{u}_{2}$

$\mathrm{v}_{2}=\frac{\left(M_{2}-M_{1}\right) u_{2}+2 M_{1} u_{1}}{M_{1}+M_{2}}=\frac{0+2 M u_{1}}{M+M}$

$\mathrm{v}_{2}=\mathrm{u}_{1}$ (initial velocity of projectile)

Thus projectile and target exchange their velocities, and continue to move in the same direction. Let projectile is moving with velocity $10 \mathrm{~m} / \mathrm{s}$ and target $5 \mathrm{~m} / \mathrm{s}$, only then they will collide $\left(\mathrm{u}_{1}>\mathrm{u}_{2}\right)$. After collision projectile does not rebound, thus suffers no reaction and there is no motion in reverse direction, and moves with reduced velocity $5 \mathrm{~m} / \mathrm{s}$. The third law of motion is not obeyed as there is no reaction (the projectile does not retrace the path).

It is again added that equations are written for ideal elastic collisions, the Reaction (final velocity of Projectile) is different from Action (initial velocity of projectile) in these cases, and thus the Third Law of Motion is inconsistent in such cases. The action and reaction for projectiles and targets of different masses in one dimension are shown in Table 4 for comparison.

\section{Generalized form of the Third Law of Motion}

It is a basic principle of science that no conclusions can be drawn on the basis of a single or few qualitative observations. If the results are repeated under all conditions for different parameters, only then the law is accepted experimentally.

On the basis of the above observations The Principia's Third Law of Motion is generalized:

'To every action there may be reaction, but May or may not be always equal and opposite; depending upon the inherent characteristics of the interacting bodies.' Or "The mutual actions of two bodies may not always be equal and opposite, depending upon the inherent characteristics of the interacting system".

As Newton elaborated the law in terms of force and velocity. But Newton's law is not fully applied to all those phenomena where it is applicable. The reason may be that Newton himself gave just three qualitative examples to elaborate the law. Further action and reaction are the devoid of units and dimensions. So the definition of the law can be expressed in terms of force and velocity separately. In such cases the effects of characteristics of the projectile and target become evident, which were veiled as Newton defined the law in terms of action and reaction. The law is defined in sub-section (2.2), analogous to Newton's law. The velocity is associated with momentum and kinetic energy, and total energy is conserved in every process. The generalized form of the third law can be expressed as

Action $\propto$ Reaction

or Reaction $=-$ K Action

$\mathrm{K}$ is the coefficient of proportionality and depends upon the inherent characteristics of interacting bodies or mode of interactions. The coefficient of proportionality takes into account the inherent characteristics, nature, composition, flexibility, elasticity, rigidity, magnitude, plasticity, distinctiveness of interacting bodies or mode of interactions, and other relevant factors. The bodies may be of steel, wood, rubber, cloth, wool, clay, kneaded flour, chewing gum, sponge, spring, etc. The bodies can be of solid, liquid, gas or mixture of all. The law is applicable for all bodies thus can be considered for waves as well. 
The coefficient of proportionality, $\mathrm{K}$ is consistent with existing coefficients in physics and can be experimentally determined for different cases. It has been discussed that action and reaction are different in many cases. Thus generalized form of third law is universally applicable, and the Principia's law is applicable for ideal or super special cases only.

There is no factor in Newton's Third Law of Motion which could affect equality of Action and Reaction. In calculation of time period of pendulum, in verification of Ohm's law, in determination of force in Coulomb's law, in many other laws etc. the external or internal conditions play a significant role. Nuclear fission is only possible for uranium not for steel. But it is not so in case of Newton's Third Law of Motion, which implies irrespective of any external or internal effect, other characteristics of bodies etc., the action and reaction must be precisely equal. So, the law is true under some conditions and action and reaction equality holds good in some cases only or under tailor made conditions. Hence, its generalization is justified, as it takes all factors in account.

\section{Conclusions}

Newton stated third law of motion in terms of action and reaction, which do not possess units and dimensions. Newton did not give any equation to quantitatively justify the law experimentally, which is the first and foremost condition for any law now days. The law is universally applicable for all bodies under all conditions. The bodies may be of steel, wood, rubber, cloth, wool, sponge, spring, typical plastic, porous material, air/fluid filled artifact, mud or kneaded flour or chewing gum specifically fabricated material etc. For all such bodies if the action is same, then the reaction must be the same. Newton did not give any mathematical equation for the law. The applications of third law of motion are logically extended and generalized form is justified experimentally.

In the Principia the law was not justified with help of mathematical equations, the explanation is qualitative not quantitative; which is absolute necessity for scientific validity of the law. While elaborating the law Newton gave three examples, and action and reaction were expressed in terms of force and velocity. Thus Newton's law is applicable when body is at rest or moves with velocity. Newton's third law can be expressed in terms of force and velocity individually, analogous to action and reaction law. The velocity is further related with momentum and kinetic energy, and energy is conserved in all processes.

As Newton defined the third law in terms force and velocity, then the law can also be equivalently defined in terms of force and velocity (as in terms of action and reaction). If so then in definition and explanation of the law importance of inherent characteristics of impinging and colliding bodies can be understood. If so then not only the third law of motion is clearly expressed but its meaning becomes more transparent. The complete critical analysis of the law implies that the third law does not take into account the significant factors e.g. Inherent characteristics, nature, compositions, flexibility, rigidity, magnitude, size, elasticity, shape, distinctiveness of interacting bodies, mode of interactions, point of impact etc. These factors can be taken in account if the law is generalized. Thus the law cannot be regarded as quantitatively true until confirmed specifically in all cases. The mathematical form of generalized law is Reaction $=-\mathrm{K}$ action, the value of $\mathrm{K}$ may be equal to, less than, or greater than unity depending upon experimental parameters. The value of $\mathrm{K}$ can be determined experimentally. As discussed in Tables 2-4, Newton's law is confirmed in only first observation, not in other observations. Thus in such cases kinetic energy is not dissipated to other forms of energy. The rest of observations can be explained on the basis of generalized form of the Third Law of motion.

Had Newton expressed this law in terms of force, velocity, momentum, energy etc., instead of action and reaction, then understanding would have much simpler and easier now. In Newton's time it was beginning of physics, due to lack of systematic scientific quantities, third law was expressed philosophically. The major reason for this is that in Newton's time units and dimensions were not discovered, and also mathematical equations (algebraic or analytical) were not used to explain the phenomena, so Newton used philosophical and geometrical methods to interpret the phenomena. But now specifically scientific terms (possessing units and dimensions) can be used to understand the law, which lead to noble ideas i.e. generalization of third law of motion.

The critical analysis or refinement in existing theories is continuous process in science when new experimental evidences emerge. The null results of Michelson Morley experiments may be regarded as the earliest experimental origin of theory of relativity which is now an established theory; even then the rudiments of Michelson Morley 
experiments are questioned by scientists and attempt is made to give alternate theory the formation of universe has replaced the Steady State Theory; but it did not resist scientists to perceive new ideas to substitute the big bang theory周, The imaginative ideas act as scientific catalysts. The theories continue to be accepted as long as they are supported by logical theoretical deductions and experimental results; it is the acid test for existing and prospective theories. When the applications of classical mechanics are extended to small particles and particles having velocities comparable to that of light, then quantum mechanics and theory of relativity are originated. The geocentric theory is replaced by heliocentric theory. Even priority of Newton's second law of motion is questioned, as $\mathrm{F}=\mathrm{ma}$ is given more clearly and transparently by Euler ${ }^{\text {10. In }}$. the current discussion, there is generalization of the third law within realm of classical mechanics. Thus Physics is not static body of doctrine but a developing science. The speculative EM drive is being put into practical prospective in various laboratories. However the logical theoretical deductions and experimental evidences are the main criteria which decide the acceptance or rejection of a theory.

\section{Acknowledgements}

The author is highly indebted to Prof. Robert Bradley, Professor B. C. Chauhan, Dr. Steve Crothers and Dr. Parvin Kumar for encouragement at various stages of the work.

\section{References}

1. Newton I. Mathematical Principles of Natural Philosophy. London; 1729. p. 19-20.

2. Fourier J. The Analytical Theory of Heat. Cambridge University Press; 1878.

3. Martins R. The origin of dimensional analysis. Journal of Franklin Institute. 1981; 311(5):331-7. Crossref

4. Rossiter W. Dictionary of Scientific Terms. London and Glasgow: William Collins Sons and Coy; 1879. p. 109.

5. Everett. First Report of the Committee for the Selection and Nomenclature of Dynamical and Electrical Units. 43rd Meeting of the British Association for the Advancement of Science; Bradford. 2012. p. 223.

6. National Council of Educational Research and Training. 2006. p. 96-7.

7. What makes a collision superelastic? 2015. Available from: Crossref

8. White H. Measurement of impulsive thrust from a closed radio-frequency cavity in vacuum. Journal of Propulsion and Power. 2017; 33(4):830-41. Crossref

9. Roger S. Browse New Scientist Magazine. 2006. p. 30.

10. Ivlev AV. Statistical mechanics where Newton's third law is broken. Physical Review X. 2015. p. 1-10. Crossref

11. Elastic collision. 2017. Available from: Crossref

12. Resnick R, Halliday D. Physics Part I. 2nd ed. New Delhi: Wiley Eastern Limited; 1996. p. 215-22.

13. Prakashan P. Acta Ciencia Indica Mathematics. 1987; 13.

14. Khan MS. Michelson-Morley experiment: A misconceived and misinterpreted experiment. Indian J Sci Technol. 2011; 4(11):1347-57. Available from: www.indjst.org

15. Khan MS. Experimental and theoretical evidences of fallacy of space-time concept and actual state of existence of the physical universe. Indian J Sci Technol. 2012; 5(3):2217-39. Available from: www.indjst.org

16. Sharma A. Physics Essays 27. 2014; 3:503-9. 